\title{
Prognostic value of preoperative peripheral blood mean platelet volume/platelet count ratio (MPV/PC) in patients with resectable cervical
}

\section{cancer}

\author{
Qicheng Deng, Qifang Long, Yanan Liu, Zhujuan Yang, Yibei Du and Xin Chen ${ }^{*}$
}

\begin{abstract}
Background: The mean platelet volume/platelet count ratio (MPV/PC) ratio based on the preoperative peripheral MPV and PCcan be used to predict the prognosis of multiple malignant tumors.

Objective: To evaluate the prognostic value of MPV/PC in cervical cancer patients.

Methods: This study enrolled 408 patients who had undergone radical surgery for cervical cancer and evaluated the correlation of MPV/PC with patient prognosis in the primary cohort and validation cohort. Additionally, independent prognostic factors were incorporated to construct the prognostic nomogram, and the area under the receiver operating characteristic (ROC) curve (AUC) value was calculated to analyze the prognostic predictive ability of the nomogram.

Results: In the primary cohort, Kaplan-Meier survival analysis indicated that the overall survival (OS) for patients with $M P V / P C \leq 0.41$ was significantly lower than that in patients with MPV/PC $>0.41$. MPV/PC was an independent prognostic factor for resectable cervical cancer patients. Compared with neutrophi//lymphocyte ratio (NLR), platelet/lymphocyte ratio (PLR) or monocyte/lymphocyte ratio (MLR), the AUC values of MPV/PC in predicting the 3- and 5-year survival rates for cervical cancer patients were greater. Similar results were verified in the validation cohort. Subsequently, the nomogram constructed based on MPV/PC, International Federation of Gynecology and Obstetrics (FIGO) classification and lymphovascular invasion performed well to accurately predict the prognosis of cervical cancer patients. The 3- and 5-year survival rates predicted by the nomogram were highly consistent with the real observations. Similar results were also displayed in the validation cohort.
\end{abstract}

Conclusions: MPV/PC may be used as a novel independent prognostic factor for patients with resectable cervical cancer. Compared with the FIGO classification system, the nomogram integrating MPV/PC maybe reliably predict the survival of cervical cancer patients after radical surgery.

Keywords: MPV/PC, Cervical cancer, Prognosis, Nomogram

*Correspondence: chenxin1978@suda.edu.cn.

Department of Obstetrics and Gynecology, The Second Affiliated Hospital

of Soochow University, 1055 Sanxiang Street, Suzhou 215004, Jiangsu

Province, China

\begin{abstract}
Introduction
The morbidity and mortality of cervical cancer rank 4th among female malignant tumors worldwide [1]. Globally, approximately 527,600 new cervical cancer cases and 265,700 cervical cancer-related deaths are reported annually [1]; among them, more than $80 \%$ of cases come
\end{abstract}


from developing countries. According to statistics, 98,900 new cervical cancer cases and 30,500 deaths occurred in China in 2015 [2]. Currently, the International Federation of Gynecology and Obstetrics (FIGO) classification system is mainly used to judge the clinical prognosis of cervical cancer. However, the FIGO classification system is restricted in judging the prognosis of cervical cancer patients. So, other indexes must be applied (such as hematological indexes, pathological type, histological grade, tumor infiltration depth or scope, and lymph node metastasis (LNM) staging) to comprehensively judge the patient prognosis.

In recent years, hematological indexes have attracted extensive attention regarding their predictive value in malignant tumors $[3,4]$. In cervical cancer, the neutrophil/lymphocyte ratio (NLR), platelet/lymphocyte ratio (PLR), monocyte/lymphocyte ratio (MLR) and systemic inflammation response index (SIRI) are closely related to the prognosis of cervical cancer patients [5]. Recent studies have indicated that the MPV/PC ratio based on the mean platelet volume (MPV) and platelet count (PC) can predict the prognosis of multiple malignant tumors [6-12]. However, the prognostic value of MPV/PC in cervical cancer has not yet been investigated. Therefore, this study aimed to explore the influencing factors for the prognosis of cervical cancer patients, evaluate the prognostic values of MPV/PC, NLR, PLR and MLR in cervical cancer, and construct a prognostic nomogram for resectable cervical cancer patients on this basis. Additionally, the prognostic prediction accuracy of this model was compared with that of the FIGO classification system to guide clinical practice and improve the clinical outcomes of cervical cancer patients.

\section{Materials and methods Clinical data of patients}

Together, 283 patients who received radical surgery for cervical cancer at the Second Affiliated Hospital of Soochow University from 2009 to 2017 were retrospectively analyzed. The inclusion criteria were as follows: 1 . patients pathologically diagnosed with primary cervical squamous carcinoma with FIGO stage IA-IIA; 2. patients with no chronic heart, liver, kidney disease, diabetes, or severe infection; 3. patients who did not take antiplatelet agents or receive anticoagulation therapy within 1 month before examination; 4 . patients with complete clinical and follow-up data; and 5. patients who did not receive neoadjuvant chemotherapy (NCT) or radiotherapy. The follow-up plan after initial treatment comprised re-examinations every 3 months within 1-2years after surgery, every 6 months within $3-5$ years and yearly thereafter. The follow-up examinations mainly included interrogation, gynecological examination, cervical cytological examination, transvaginal ultrasonography, and CT or MRI. Overall survival (OS) was defined as the time from the date of surgery to the date of death from any cause or the last follow-up for the survivors or those lost to follow-up (censor). The last follow-up was conducted on June 30th, 2019, and the median follow-up period was 72 (range, 4-129) months. Additionally, 125 cervical cancer patients who had undergone radical resection at the Kunshan First People's Hospital were recruited as the validation cohort. The study design conformed to the Declaration of Helsinki and was approved by the Ethics Committee of the Second Affiliated Hospital of Soochow University (2009-KY-043). All the patients provided written informed consent.

\section{Data collection}

The clinical information, clinicopathological parameters and preoperative routine blood tests of the patients were collected. Clinical information included patient age, FIGO stage, postoperative therapeutic scheme, smoking history, medical complications and surgical

Table 1 Clinicopathological characteristics of patients with cervical cancer in primary cohort and validation cohort

\begin{tabular}{|c|c|c|c|c|}
\hline \multirow[t]{2}{*}{ Characteristic } & \multicolumn{2}{|c|}{ Primary Cohort $(n=283)$} & \multicolumn{2}{|c|}{$\begin{array}{l}\text { Validation Cohort } \\
(n=125)\end{array}$} \\
\hline & No. of Patients & $\%$ & $\begin{array}{l}\text { No. of } \\
\text { Patients }\end{array}$ & $\%$ \\
\hline \multicolumn{5}{|l|}{ Age } \\
\hline$\leq 45$ & 136 & 48.1 & 62 & 49.6 \\
\hline$>45$ & 147 & 51.9 & 63 & 50.4 \\
\hline \multicolumn{5}{|c|}{ Histological grade } \\
\hline G1 & 22 & 7.8 & 12 & 9.2 \\
\hline G2 & 147 & 51.9 & 67 & 53.6 \\
\hline G3 & 114 & 40.3 & 46 & 36.8 \\
\hline \multicolumn{5}{|c|}{ Tumor invasion depth } \\
\hline$\leq 1 / 2$ & 219 & 77.4 & 72 & 57.6 \\
\hline$>1 / 2$ & 64 & 22.6 & 53 & 42.4 \\
\hline \multicolumn{5}{|l|}{ Tumor size } \\
\hline$\leq 4$ & 155 & 54.8 & 78 & 60.0 \\
\hline$>4$ & 128 & 45.2 & 52 & 40.0 \\
\hline \multicolumn{5}{|c|}{ Lymphovascular invasion } \\
\hline No & 233 & 82.3 & 87 & 69.6 \\
\hline Yes & 50 & 17.7 & 38 & 30.4 \\
\hline \multicolumn{5}{|l|}{ FIGO stage } \\
\hline IA & 65 & 23.0 & 31 & 24.8 \\
\hline $\mathrm{IB}$ & 166 & 58.7 & 59 & 47.2 \\
\hline$\| \mathrm{A}$ & 52 & 18.4 & 35 & 28.0 \\
\hline \multicolumn{5}{|l|}{ Radiotherapy } \\
\hline No & 241 & 85.2 & 90 & 72.0 \\
\hline Yes & 42 & 14.8 & 35 & 28.0 \\
\hline
\end{tabular}


complications. Pathological features mainly included pelvic lymph node (PLN) metastasis status, tumor size, histological grade, tumor invasion depth, lymphovascular invasion and surgical margin. The routine blood tests at 1 week before surgery included those to evaluate $\mathrm{WBC}, \mathrm{Hb}$, monocytes, neutrophils, lymphocytes, PC, MPV and the platelet distribution width (PDW), along with NLR, PLR, MLR and MPV/PC calculated based on the abovementioned results. Additionally, the optimal cutoff values of some continuous variables were determined by the receiver operating characteristic (ROC) curve to convert them into categorical variables [12].

\section{Statistical analysis}

The intergroup differences in variable data were analyzed by chi-squared test or t-test. The Kaplan-Meier curve was adopted for survival analysis and tested using the log-rank test. To evaluate the discriminating ability of MPV/PC, NLR, PLR and MLR in cervical cancer prognosis, ROC curves were plotted to preliminarily evaluate the area under the curve (AUC). Next, the prognostic factors for patients were evaluated using the univariate Cox proportional hazard regression model. Later, the multivariate Cox proportional hazard model was constructed using the reverse step-by-step deletion method. Afterward, the variables selected by the multivariate

Table 2 Correlations between preoperative SII and clinicopathological characteristics in primary and validation cohort

\begin{tabular}{|c|c|c|c|c|c|c|c|c|}
\hline \multirow[t]{2}{*}{ Clinical parameter } & \multicolumn{4}{|l|}{ Primary Cohort } & \multicolumn{4}{|c|}{ Validation Cohort } \\
\hline & $\begin{array}{l}M P V / P C \leq 0.41 \\
(141)\end{array}$ & $\begin{array}{l}\text { MPV/PC }>0.41 \\
(142)\end{array}$ & $x^{2}$ & $P$ & $\begin{array}{l}\text { MPV/ } \\
P C \leq 0.41(55)\end{array}$ & $\begin{array}{l}\text { MPV/ } \\
\text { PC>0.41(70) }\end{array}$ & $x^{2}$ & $P$ \\
\hline Age & & & 0.09 & 0.768 & & & 1.40 & 0.237 \\
\hline$\leq 45$ & 69 & 67 & & & 24 & 38 & & \\
\hline$>45$ & 72 & 75 & & & 31 & 32 & & \\
\hline Histological grade & & & 0.33 & 0.850 & & & 0.30 & 0.860 \\
\hline G1 & 12 & 10 & & & 5 & 7 & & \\
\hline G2 & 74 & 73 & & & 31 & 36 & & \\
\hline G3 & 55 & 59 & & & 19 & 27 & & \\
\hline Tumor invasion depth & & & 0.78 & 0.376 & & & 0.01 & 0.907 \\
\hline$\leq 1 / 2$ & 106 & 113 & & & 32 & 40 & & \\
\hline$>1 / 2$ & 35 & 29 & & & 23 & 30 & & \\
\hline Tumor size & & & 0.01 & 0.957 & & & 1.48 & 0.223 \\
\hline$\leq 4$ & 77 & 78 & & & 27 & 42 & & \\
\hline$>4$ & 64 & 64 & & & 28 & 28 & & \\
\hline Lymphovascular invasion & & & 0.12 & 0.734 & & & 2.81 & 0.094 \\
\hline No & 115 & 118 & & & 34 & 53 & & \\
\hline Yes & 26 & 24 & & & 21 & 17 & & \\
\hline FIGO stage & & & 3.51 & 0.173 & & & 0.58 & 0.749 \\
\hline IA & 39 & 26 & & & 14 & 17 & & \\
\hline IB & 78 & 88 & & & 24 & 35 & & \\
\hline$\| A$ & 24 & 28 & & & 17 & 18 & & \\
\hline Radiotherapy & & & 2.71 & 0.099 & & & 0.32 & 0.574 \\
\hline No & 125 & 116 & & & 41 & 49 & & \\
\hline Yes & 16 & 26 & & & 14 & 21 & & \\
\hline NLR & & & 0.18 & 0.674 & & & 1.73 & 0.189 \\
\hline$N L R \leq 2.75$ & 77 & 74 & & & 18 & 31 & & \\
\hline$N L R>2.75$ & 64 & 68 & & & 37 & 39 & & \\
\hline PLR & & & 7.26 & $0.007^{*}$ & & & 17.96 & $<0.001^{*}$ \\
\hline$P L R \leq 128$ & 52 & 75 & & & 21 & 53 & & \\
\hline PLR > 128 & 89 & 67 & & & 34 & 17 & & \\
\hline MLR & & & 1.36 & 0.243 & & & 0.54 & 0.462 \\
\hline$M L R \leq 0.27$ & 90 & 81 & & & 28 & 31 & & \\
\hline$M L R>0.27$ & 51 & 61 & & & 27 & 39 & & \\
\hline
\end{tabular}



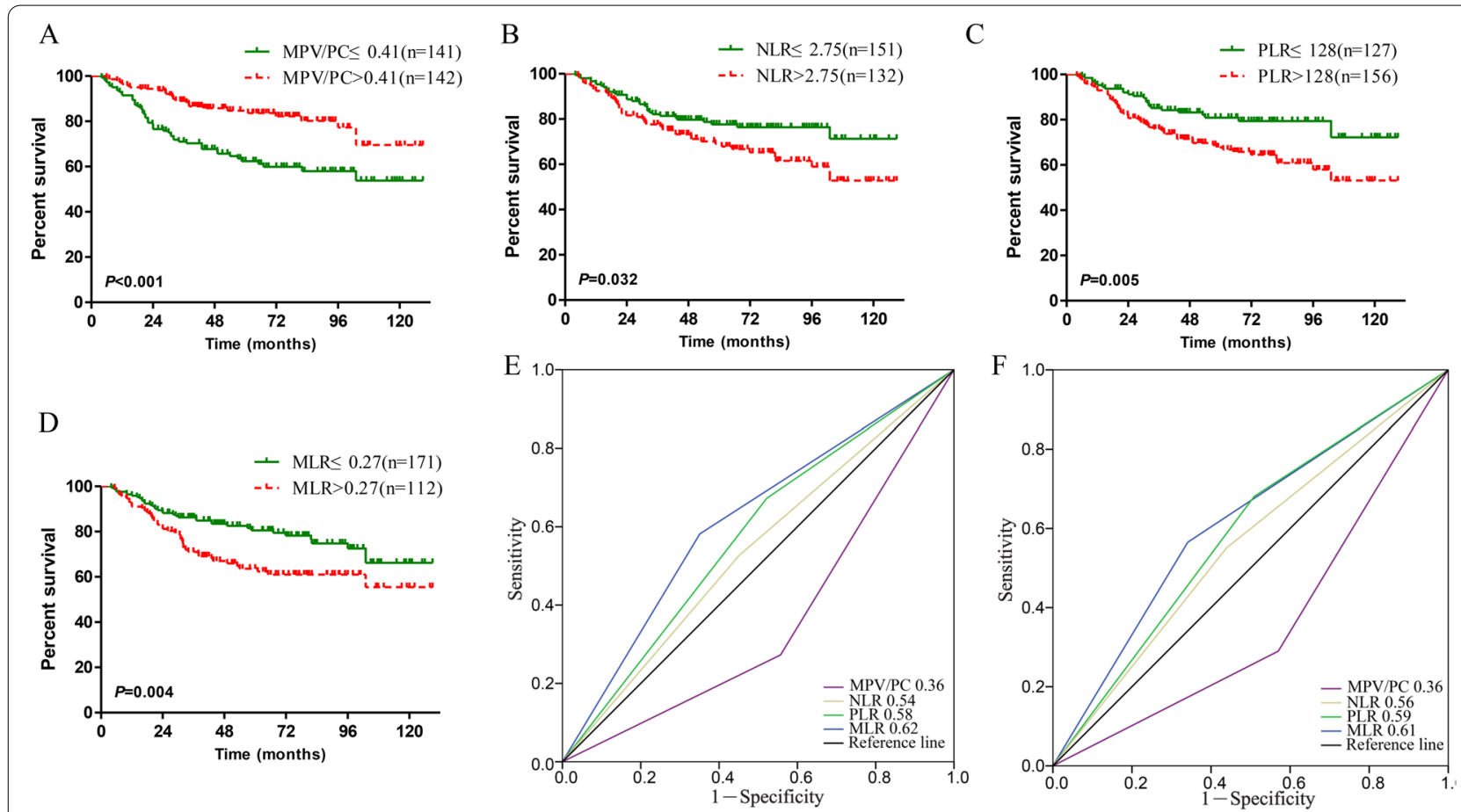

Fig. 1 Kaplan-Meier curves for cervical cancer patients stratified by (A) MPV/PC, B NLR, C PLR and D MLR in the primary cohort. The predictive ability of MPV/PC in cervical cancer patients was compared with NLR, PLR and MLR using ROC curves at 3 years $(\mathbf{E})$ and 5 years $(\mathbf{F})$ in the primary cohort

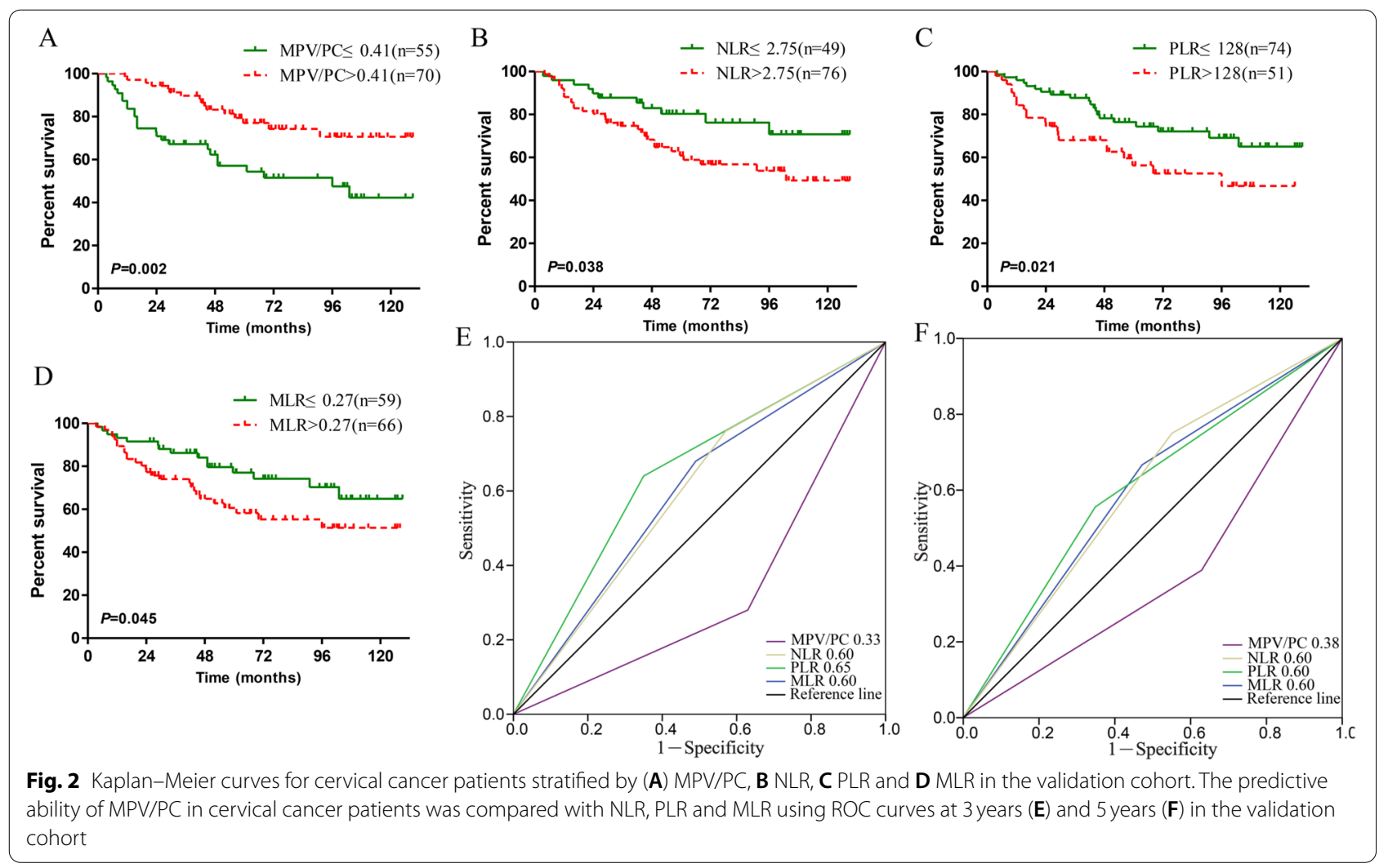


Cox model were used to construct the nomogram. Typically, the C-index and AUC were mainly used to assess the performance of the nomogram. Bootstrap sampling was repeated 1000 times for nomogram verification and evaluation calibration. The rms module of the $\mathrm{R}$ programming language, SPSS 22.0 and GraphPad Prism 5 were employed for statistical analysis. All $p$ values were two-sided, and a difference of $P<0.05$ indicated statistical significance.

\section{Results}

\section{Patient characteristics}

In total, 408 cervical cancer patients who had undergone radical surgery were enrolled in this study. The clinicopathological features of patients in the primary cohort $(n=283)$ and validation cohort $(n=125)$ are displayed in Table 1. In the primary cohort, the median patient age was 47 (range, 24-84) years. In the validation group, the median follow-up period was 47 (range, 3-120) months, and the median patient age was 43 (range, 24-67) years. The optimal cut-off value based on the primary cohort was were selected by ROC curve analysis as follows: MPV/PC (MPV/PC $\leq 0.41, \mathrm{MPV} / \mathrm{PC}>0.41)$, NLR $(\mathrm{NLR} \leq 2.75, \quad \mathrm{NLR}>2.75), \quad$ PLR $\quad(\mathrm{PLR} \leq 128, \quad \mathrm{PLR}>128)$ and MLR $(M L R \leq 0.27$, MLR > 0.27). The clinicopathological features of patients in the two cohorts were comparable, except for the tumor invasion depth. The correlations of MPV/PC with the clinicopathological features of cervical cancer patients are displayed in Table 2. In the primary cohort, MPV/PC was only negatively correlated with PLR $(P=0.007)$ but not related to other pathological parameters. Similar results were also obtained in the validation cohort.

\section{Survival analysis}

In the primary cohort, the Kaplan-Meier survival curves of the MPV/PC, NLR, PLR and MLR indices are presented in Fig.1A-D. The OS of patients with MPV/ $\mathrm{PC} \leq 0.41$ was significantly lower than that of patients with $\mathrm{MPV} / \mathrm{PC}>0.41 \quad(P<0.001 ;$ Fig. $1 \mathrm{~A})$. Additionally,

Table 3 Univariate and multivariate cox regression analyses for overall survival in cervical cancer patients in primary cohort

\begin{tabular}{|c|c|c|c|c|}
\hline \multirow[t]{2}{*}{ Variables } & \multicolumn{2}{|c|}{ Univariate analysis } & \multicolumn{2}{|c|}{ Multivariate analysis } \\
\hline & $\mathrm{HR}(95 \% \mathrm{Cl})$ & $P$ value & $\mathrm{HR}(95 \% \mathrm{Cl})$ & $P$ value \\
\hline \multicolumn{5}{|l|}{ Age } \\
\hline$\leq 45$ years vs. $>45$ years & $0.99(0.64-1.55)$ & 0.984 & & \\
\hline Histological grade & & 0.228 & & \\
\hline G1 & Ref. & - & & \\
\hline G2 & $1.01(0.43-2.81)$ & 0.486 & & \\
\hline G3 & $1.60(0.63-4.05)$ & 0.325 & & \\
\hline \multicolumn{5}{|l|}{ Tumor invasion depth } \\
\hline$>1 / 2$ vs. $\leq 1 / 2$ & $1.79(1.11-2.87)$ & $0.017^{*}$ & $0.99(0.57-1.71)$ & 0.970 \\
\hline \multicolumn{5}{|l|}{ Tumor size } \\
\hline$>4$ vs. $\leq 4$ & $2.10(1.33-3.30)$ & $0.001^{*}$ & $1.25(0.75-2.08)$ & 0.397 \\
\hline \multicolumn{5}{|l|}{ Lymphovascular invasion } \\
\hline Yes vs. No & $2.11(1.30-3.43)$ & $0.003^{*}$ & $2.27(1.38-3.73)$ & $0.001^{*}$ \\
\hline FIGO stage & & $<0.001^{*}$ & & $<0.001^{*}$ \\
\hline IA & Ref. & & Ref. & \\
\hline IB & $3.37(1.44-7.89)$ & $0.005^{*}$ & $3.85(1.60-9.29)$ & $0.003^{*}$ \\
\hline$\| A$ & $8.18(3.37-19.86)$ & $<0.001^{*}$ & $10.16(3.69-27.98)$ & $<0.001^{*}$ \\
\hline \multicolumn{5}{|l|}{ Radiotheropy } \\
\hline Yes vs. No & $0.70(0.35-1.40)$ & 0.312 & & \\
\hline \multicolumn{5}{|l|}{ MPV/PC } \\
\hline$>0.41$ vs. $\leq 0.41$ & $0.42(0.26-0.67)$ & $<0.001^{*}$ & $0.32(0.19-0.51)$ & $<0.001^{*}$ \\
\hline \multicolumn{5}{|l|}{ NLR } \\
\hline$>2.75$ vs. $\leq 2.75$ & $1.62(1.04-2.54)$ & $0.034^{*}$ & $1.53(0.97-2.43)$ & 0.070 \\
\hline \multicolumn{5}{|l|}{ PLR } \\
\hline$>128$ vs. $\leq 128$ & $1.96(1.22-3.15)$ & $0.006^{*}$ & $1.57(0.92-2.68)$ & 0.096 \\
\hline \multicolumn{5}{|l|}{ MLR } \\
\hline$>0.27$ vs. $\leq 0.27$ & $1.83(1.18-2.85)$ & $0.007^{*}$ & $1.50(0.90-2.51)$ & 0.122 \\
\hline
\end{tabular}

MPV/PC mean platelet volume/platelet count, NLR neutrophil lymphocyte ratio, $P L R$ platelet lymphocyte ratio, $M L R$ monocyte lymphocyte ratio 
the NLR, PLR and MLR indices may be used to evaluate the prognosis of cervical cancer patients, and the differences were statistically significant (Fig. 1B-D). Subsequently, the prognostic values of the 4 indices were compared using the AUC value. Compared with other systemic inflammatory indices, the MPV/PC index displayed greater AUC values in predicting the 3 - and 5-year survival rates for cervical cancer patients, indicating that MPV/PC had better prognostic value than NLR, PLR or MLR for cervical cancer patients (Fig. 1E-F). Additionally, similar results were also obtained in the validation cohort (Fig. 2A-F).

In the primary cohort, the univariate Cox proportional hazard regression model indicated that tumor invasion depth, tumor size, lymphovascular invasion, FIGO stage, MPV/PC, NLR, PLR and MLR were prognostic factors for cervical cancer patients (Table 3). The multivariate Cox proportional hazard regression model indicated that lymphovascular invasion, FIGO stage and MPV/PC were independent prognostic factors for cervical cancer patients (Table 3). In the validation cohort, tumor invasion depth was not significantly related to the prognosis of cervical cancer patients, a finding that might be related to the small sample size. The other results were consistent with those obtained in the primary cohort (Table 4).

\section{Construction and validation of the nomogram}

In the primary cohort, independent risk factors for cervical cancer, including MPV/PC, FIGO stage and lymphovascular invasion, were used to construct nomogram models to predict the 3- and 5-year OS of cervical cancer patients (Fig. 3). The C-index of the as-constructed nomogram was 0.77 , significantly higher than the 0.68 of the FIGO stage $(P<0.001)$. AUC analysis revealed that the AUC value of the nomogram was significantly greater than that of the FIGO stage (Fig. 4A and B), indicating that the nomogram might be used to assess the prognosis for patients with resectable cervical cancer and that it was more accurate than the traditional FIGO stage. In internal verification, the calibration curve of the

Table 4 Univariate and multivariate cox regression analyses for overall survival in cervical cancer patients in validation cohort

\begin{tabular}{|c|c|c|c|c|}
\hline \multirow[t]{2}{*}{ Variables } & \multicolumn{2}{|l|}{ Univariate analysis } & \multicolumn{2}{|c|}{ Multivariate analysis } \\
\hline & $\mathrm{HR}(95 \% \mathrm{Cl})$ & $P$ value & $\mathrm{HR}(95 \% \mathrm{Cl})$ & $P$ value \\
\hline \multicolumn{5}{|l|}{ Age } \\
\hline$\leq 45$ years vs. $>45$ years & $1.48(0.80-2.72)$ & 0.212 & & \\
\hline \multicolumn{5}{|l|}{ Histological grade 0.859} \\
\hline G1 & Ref. & - & & \\
\hline G2 & $1.27(0.44-3.68)$ & 0.660 & & \\
\hline G3 & $1.10(0.365-3.32)$ & 0.865 & & \\
\hline \multicolumn{5}{|l|}{ Tumor invasion depth } \\
\hline$>1 / 2$ vs. $\leq 1 / 2$ & $1.58(0.86-2.89)$ & 0.142 & & \\
\hline \multicolumn{5}{|l|}{ Tumor size } \\
\hline$>4$ vs. $\leq 4$ & $2.03(1.10-3.74)$ & $0.024^{*}$ & $1.29(0.67-2.52)$ & 0.449 \\
\hline \multicolumn{5}{|l|}{ Lymphovascular invasion } \\
\hline Yes vs. No & $2.20(1.19-4.07)$ & $0.012^{*}$ & $2.63(1.34-5.13)$ & $0.005^{*}$ \\
\hline FIGO stage & & $0.001^{*}$ & & $<0.001^{*}$ \\
\hline IA & Ref. & & Ref. & \\
\hline $\mathrm{IB}$ & $6.13(1.42-26.43)$ & $0.015^{*}$ & $7.91(1.79-34.92)$ & $0.006^{*}$ \\
\hline$\| A$ & $13.16(3.08-56.19)$ & $0.001^{*}$ & $20.43(4.46-93.65)$ & $<0.001^{*}$ \\
\hline \multicolumn{5}{|l|}{ Radiotheropy } \\
\hline Yes vs. No & $1.42(0.74-2.71)$ & 0.281 & & \\
\hline \multicolumn{5}{|l|}{ MPV/PC } \\
\hline$>0.41$ vs. $\leq 0.41$ & $0.38(0.21-0.72)$ & $0.003^{*}$ & $0.35(0.18-0.66)$ & $0.001^{*}$ \\
\hline \multicolumn{5}{|l|}{ NLR } \\
\hline$>2.75$ vs. $\leq 2.75$ & $2.04(1.03-4.06)$ & $0.042^{*}$ & $1.96(0.98-3.94)$ & 0.058 \\
\hline \multicolumn{5}{|l|}{ PLR } \\
\hline$>128$ vs. $\leq 128$ & $2.02(1.10-3.71)$ & $0.024^{*}$ & $1.74(0.92-3.31)$ & 0.090 \\
\hline \multicolumn{5}{|l|}{ MLR } \\
\hline$>0.27$ vs. $\leq 0.27$ & $1.89(1.00-3.55)$ & $0.049^{*}$ & $1.57(0.81-3.04)$ & 0.184 \\
\hline
\end{tabular}

MPV/PC mean platelet volume/platelet count, NLR neutrophil lymphocyte ratio, $P L R$ platelet lymphocyte ratio, $M L R$ monocyte lymphocyte ratio 


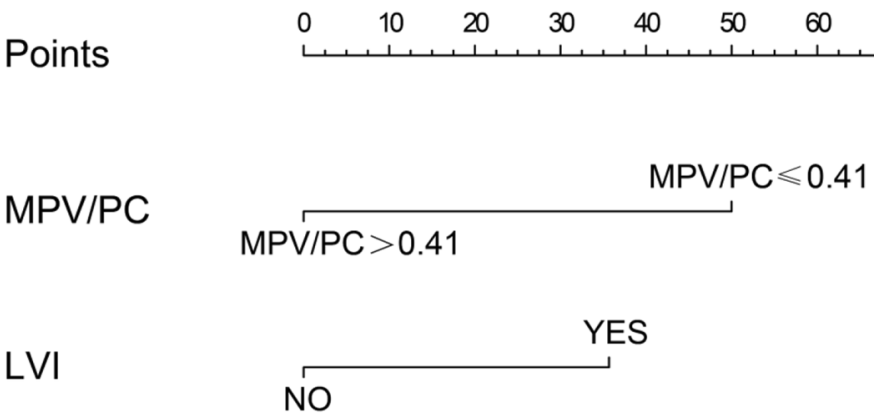

FIGO

IB

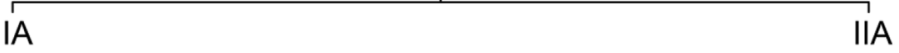

Total points

\begin{tabular}{lllllllllll}
\hline 0 & 20 & 40 & 60 & 80 & 100 & 120 & 140 & 160 & 180 & 200
\end{tabular}

3-year survival

\begin{tabular}{lllllllllll}
\hline & 0.9 & 0.85 & 0.80 .750 .7 & 0.6 & 0.5 & 0.4 & 0.3 & 0.2
\end{tabular}

\section{5-year survival}

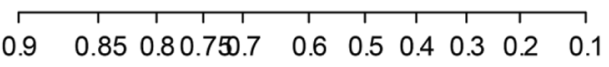

Fig. 3 Nomogram based on MPV/PC, LVI and FIGO in cervical cancer

model displayed favorable consistency between the predicted and actual values (Fig. 4C-D), demonstrating the extremely reliable repeatability of the nomogram. Furthermore, external verification of the nomogram was conducted using the validation cohort data. The C-index of the nomogram was 0.82 , which was significantly higher than the 0.72 of FIGO stage $(P<0.001)$. The AUC value of the nomogram was apparently higher than that of the FIGO classification system (Fig. 5A and B). Additionally, the calibration curve of the nomogram showed good consistency between the predicted 3-/5-year survival rates and actual observed values (Fig. $5 \mathrm{C}$ and $\mathrm{C}$ ). The above results suggested that the nomogram might serve as a tool to predict the survival of patients more effectively and accurately with resectable cervical cancer.

\section{Discussion}

Since scholars first reported the increased platelet count in cancer patients in 1872 [13], the platelet changes in blood from patients with malignant tumors at different sites have been successively investigated. Platelets are one of the bioactive visible blood components produced by mature megakaryocytes in bone marrow and affect the inflammatory response and immune regulation in the body $[14,15]$. Platelets extensively exist in the peripheral blood circulation and exert their role in tumor invasion, hematogenous metastasis and distal organ colonization through surface molecules or secreting related factors $[16,17]$. PC and MPV are the two most important indices to evaluate platelet function, and their combination in the MPV/PC index has recently been used in the prognosis evaluation of malignant tumor patients. MPV/ PC was first used as the prediction index for long-term mortality after myocardial infarction [18]. Recently, Cho et al. revealed the expression level and diagnostic value of MPV/PC in hepatocellular carcinoma (HCC) patients [6]. Subsequently, Gu et al. found that the OS of HCC patients with high MPV/PC was significantly poorer [11]. In lung carcinoma, Inagaki et al. found that MPV was expressed at low levels in lung carcinoma patients, while PC was highly expressed. The OS of non-small cell lung cancer (NSCLC) patients with low MPV/PC was significantly superior to that of patients with high MPV/PC, and $\mathrm{MPV} / \mathrm{PC}$ was an independent prognostic factor for locally advanced NSCLC [7]. In colorectal cancer (CRC), the low MPV/PC in patients suggests a low TNM stage and less LNM [9]. We confirmed that cervical cancer patients with high MPV/PC had a poor prognosis, and MPV/PC 

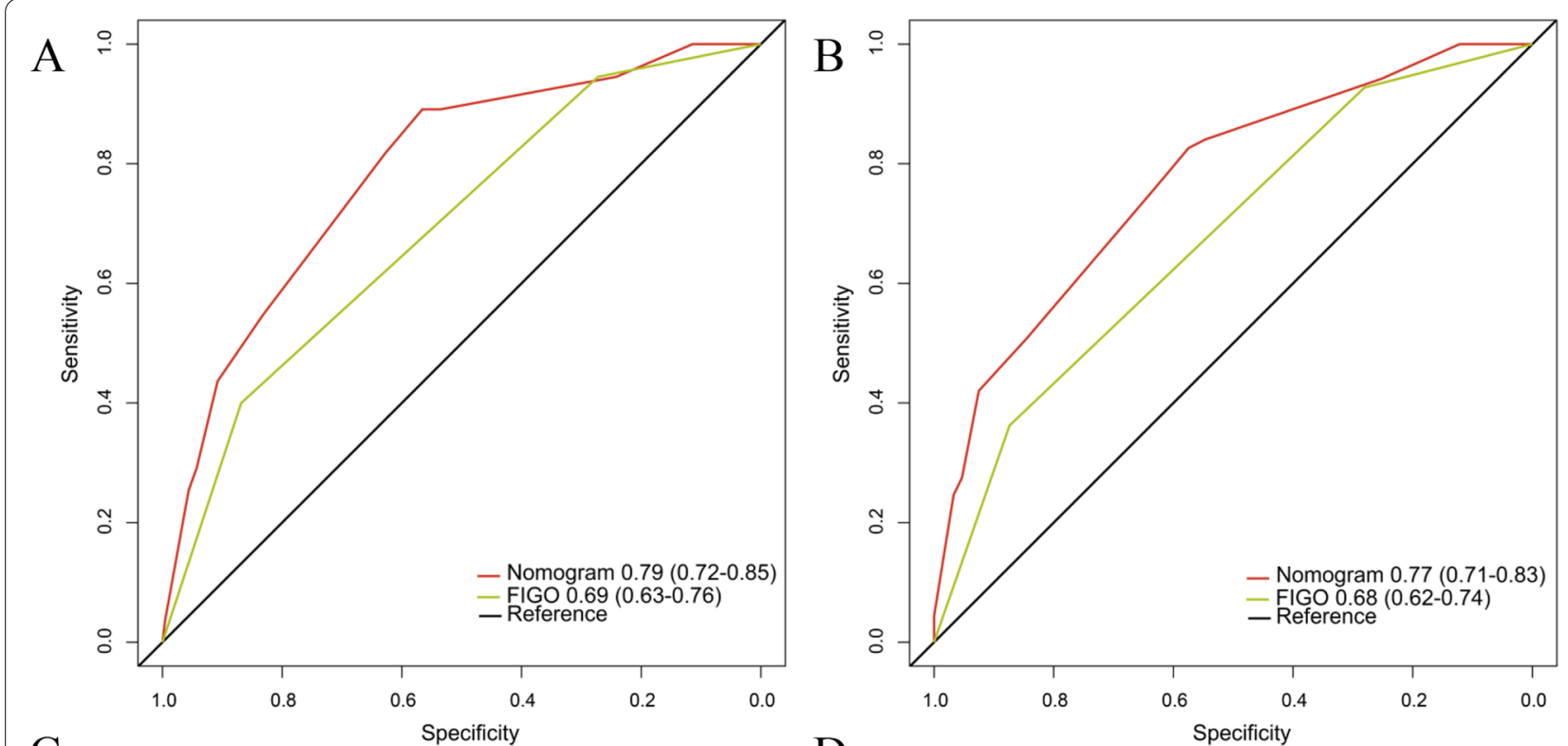

C

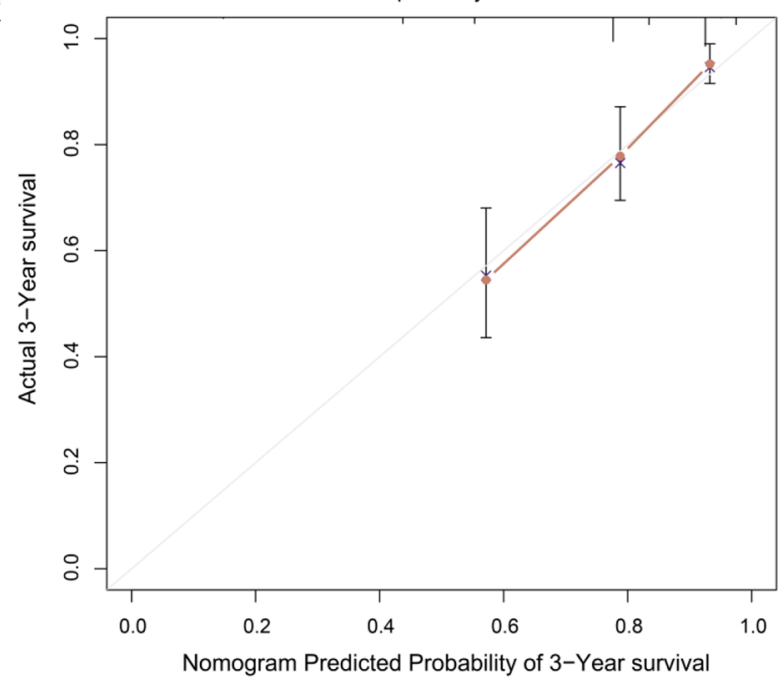

$\mathrm{D}$

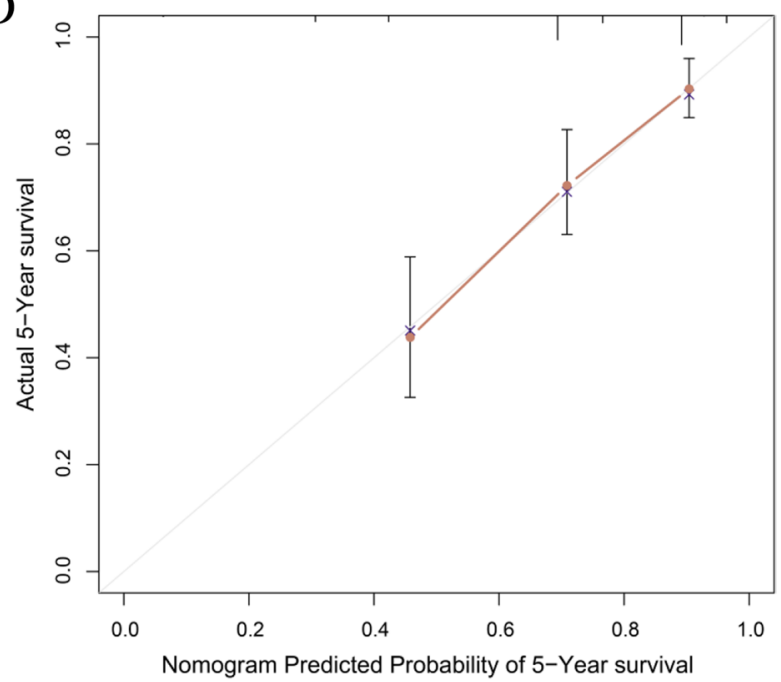

Fig. 4 The 3-year survival rate $(\mathbf{A})$ and 5-year survival rate $(\mathbf{B})$ of cervical cancer patients predicted by the nomogram were highly consistent with the actual observed values in the primary cohort. Ability of the ROC analysis nomogram to predict the 3-year survival rate (C) and 5-year survival rate (D) of cervical cancer patients. The nomogram had a larger AUC than FIGO staging in the primary cohort

was an independent prognostic factor for cervical cancer patients. The ROC curve revealed that MPV/PC showed higher prognostic value than NLR, PLR and MLR in cervical cancer. Afterward, multivariate analysis identified the clinicopathological variables MPV/PC, FIGO stage and lymphovascular invasion, which were incorporated to construct the nomogram. The nomogram exhibited a high accuracy in predicting survival $(C$-index $=0.78)$ and a significantly higher predictive ability in the survival of the primary cohort than the FIGO classification system. These results were then verified by a group of independent external verification cohort data. MPV/PC may serve as a prognostic and therapeutic marker that contributes to the early formulation of a more accurate and timelier individualized therapeutic scheme.

Malignant tumors may result in alterations in platelet parameters, but the mechanism by which MPV/ $\mathrm{PC}$ can be used to predict the prognosis of malignant tumors has not yet been completely illustrated and may be related to the following factors. Bone marrow hyperplasia is active in malignant tumor patients, tumor cells produce thrombogenic factors, and the body fluid environment concentration that promotes the generation of bone marrow megakaryocytes is elevated in the blood 

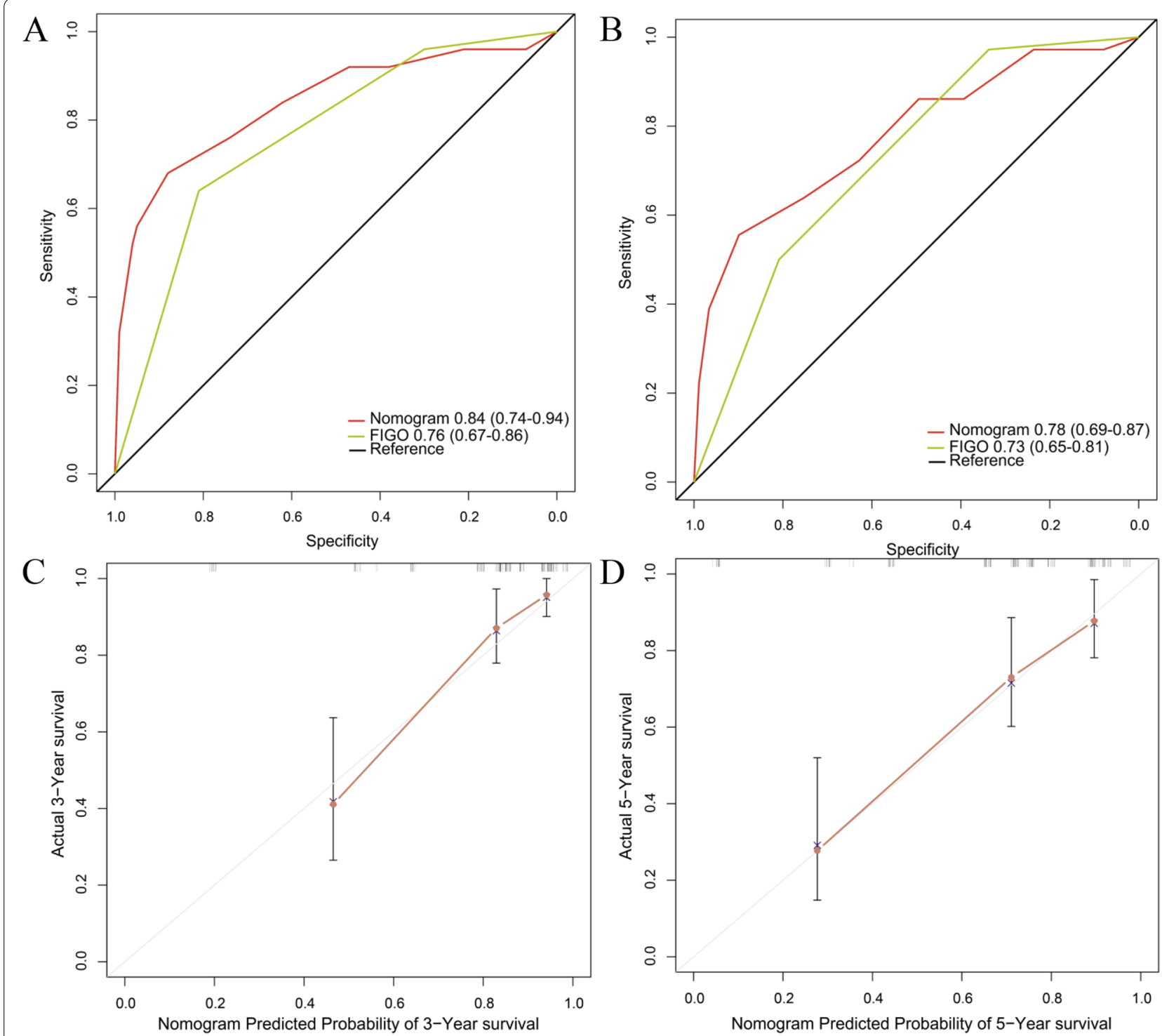

Fig. 5 The 3-year survival rate (A) and 5-year survival rate (B) of cervical cancer patients predicted by the nomogram were highly consistent with the actual observed values in the validation cohort. Ability of the ROC analysis nomogram to predict the 3-year survival rate (C) and 5-year survival rate (D) of cervical cancer patients. The nomogram had a larger AUC than FIGO staging in the validation cohort

circulation [15]. The number of cytokines that promote tumor growth is increased in tumor patients. Additionally, these cytokines, such as interleukin (IL)-1, IL-3, IL-6, IL-17, IL-18 and tumor necrosis factor- $\alpha$ (TNF$\alpha)$, specifically stimulate increased production of platelets, $[19,20]$. Stone et al. revealed that IL-6 promotes the secretion of more thrombogenic factors by the liver, while ovarian cancer cells secrete IL-6, which causes an increased amount of thrombogenic factors to act on the bone marrow, resulting in increased platelet production and a changed platelet morphology [21]. Furthermore, malignant tumor cells secrete granulocyte macrophage-colony stimulating factors, thus accelerating megakaryocyte production and thrombosis [15]. Malignant tumors consume a large amount of patient energy; thus, tumor patients may develop chronic blood loss, malnutrition and tissue necrosis, which affect platelet morphological parameters [15]. Malignant tumor cells can secrete and stimulate increased platelets to release transforming growth factor (TGF), directly stimulating the growth of certain tumor cells; however, proliferating tumor tissues produce more stimulating factors to accelerate bone marrow megakaryocyte production, thus forming a vicious cycle [15]. 
This study has certain limitations. (1) This study was a retrospective single-center study, which might be associated with selection bias. (2) Heterogeneity existed in the treatments that patients received after surgical resection, likely affecting different clinical outcomes. (3) The cutoff values in this study were not verified in other cohorts. Thus, more large-scale, multicenter clinical studies are warranted to verify our research results.

\section{Conclusion}

As a noninvasive, lost-cost, simple and repeatable index, $\mathrm{MPV} / \mathrm{PC}$ is a novel independent prognostic index for patients with resectable cervical cancer. Compared with the traditional FIGO classification system, the nomogram that integrates MPV/PC maybe reliably predict the survival of cervical cancer patients after radical surgery.

\section{Acknowledgments}

None.

\section{Authors' contributions}

Q.C.D. and X.C. conceived and designed the study and helped to draft the manuscript. Q.F.L. and Y.N.L. performed the data collection. Z.J.Y. and Y.B.D. performed the statistical analysis. All the authors have read and critically revised the manuscript for intellectual content and have approved the final manuscript.

\section{Funding}

This study was supported by Sanitation Bureau Guidance Project of Suzhou (SYSD2020088).

\section{Availability of data and materials}

The dataset supporting the conclusions of this article is available at The Second Affiliated Hospital of Soochow University from the corresponding author on reasonable request.

\section{Declarations}

\section{Ethics approval and consent to participate}

This study was carried out in accordance with the principles of the Declaration of Helsinki, and approval was obtained from the institutional review boards at the Ethics Committee of The Second Affiliated Hospital of Soochow University (2009-KY-043). Written informed consent was obtained from all patients included in the study.

\section{Consent for publication}

Not applicable.

\section{Competing interests}

The authors declare no conflicts of interest regarding the publication of this article.

Received: 25 June 2021 Accepted: 15 November 2021

Published online: 29 November 2021

\section{References}

1. Torre LA, Bray F, Siegel RL, Ferlay J, Lortet-Tieulent J, Jemal A. Global cancer statistics, 2012. CA Cancer J Clin. 2015;65(2):87-108.

2. Chen W, Zheng R, Baade PD, Zhang S, Zeng H, Bray F, et al. Cancer statistics in China, 2015. CA Cancer J Clin. 2016:66(2):115-32.
3. Mantovani A, Allavena P, Sica A, Balkwill F. Cancer-related inflammation. Nature. 2008;454(7203):436-44.

4. Shao Y, Ning Z, Chen J, Geng Y, Gu W, Huang J, et al. Prognostic nomogram integrated systemic inflammation score for patients with esophageal squamous cell carcinoma undergoing radical esophagectomy. Sci Rep. 2015;5:18811.

5. Chao B, Ju X, Zhang L, Xu X, Zhao Y. A novel prognostic marker systemic inflammation response index (SIRI) for operable cervical Cancer patients. Front Oncol. 2020;10:766

6. Cho SY, Yang JJ, You E, Kim BH, Shim J, Lee HJ, et al. Mean platelet volume/platelet count ratio in hepatocellular carcinoma. Platelets. 2013;24(5):375-7.

7. Inagaki N, Kibata K, Tamaki T, Shimizu T, Nomura S. Prognostic impact of the mean platelet volume/platelet count ratio in terms of survival in advanced non-small cell lung cancer. Lung Cancer. 2014;83(1):97-101.

8. Omar M, Tanriverdi O, Cokmert S, Oktay E, Yersal O, Pilanci KN, et al. Role of increased mean platelet volume (MPV) and decreased MPV/platelet count ratio as poor prognostic factors in lung cancer. Clin Respir J. 2018;12(3):922-9.

9. Wu YY, Zhang X, Qin YY, Qin JQ, Lin FQ. Mean platelet volume/platelet count ratio in colorectal cancer: a retrospective clinical study. BMC Cancer. 2019;19(1):314

10. Feng JF, Sheng C, Zhao Q, Chen P. Prognostic value of mean platelet volume/platelet count ratio in patients with resectable esophageal squamous cell carcinoma: a retrospective study. PeerJ. 2019;7:e7246.

11. Gu J, Zhang X, Wang Z, Cui R, Zhang J, Jia Y, et al. Simplified nomograms based on platelet-associated models for survival prediction in Asian hepatocellular carcinoma patients after surgery. Surg Oncol. 2019;30:131-8.

12. Zhang $X$, Qin YY, Chen M, Wu YY, Lin FQ. Combined use of mean platelet volume/platelet count ratio and platelet distribution width to distinguish between patients with nasopharyngeal carcinoma, those with benign tumors of the Nasopharynx, and healthy subjects. Cancer Manag Res. 2019;11:10375-82.

13. Riess H, Braun G, Brehm G, Hiller E. Critical evaluation of platelet aggregation in whole human blood. Am J Clin Pathol. 1986;85(1):50-6.

14. Cho SY, Jeon YL, Kim W, Kim WS, Lee HJ, Lee WI, et al. Mean platelet volume and mean platelet volume/platelet count ratio in infective endocarditis. Platelets. 2014;25(8):559-61.

15. Franco AT, Corken A, Ware J. Platelets at the interface of thrombosis, inflammation, and cancer. Blood. 2015;126(5):582-8.

16. Li N. Platelets in cancer metastasis: to help the "villain" to do evil. Int J Cancer. 2016:138(9):2078-87.

17. Janowska-Wieczorek A, Wysoczynski M, Kijowski J, Marquez-Curtis L, Machalinski B, Ratajczak J, et al. Microvesicles derived from activated platelets induce metastasis and angiogenesis in lung cancer. Int $\mathrm{J}$ Cancer 2005:113(5):752-60.

18. Azab B, Torbey E, Singh J, Akerman M, Khoueiry G, McGinn JT, et al. Mean platelet volume/platelet count ratio as a predictor of long-term mortality after non-ST-elevation myocardial infarction. Platelets. 2011;22(8):557-66.

19. Burger JA, Kipps TJ. CXCR4: a key receptor in the crosstalk between tumor cells and their microenvironment. Blood. 2006;107(5):1761-7.

20. Nagata S, Fukunaga R. Granulocyte colony-stimulating factor receptor and its related receptors. Growth Factors. 1993:8(2):99-107.

21. Stone RL, Nick AM, McNeish IA, Balkwill F, Han HD, Bottsford-Miller J, et al. Paraneoplastic thrombocytosis in ovarian cancer. N Engl J Med. 2012;366(7):610-8.

\section{Publisher's Note}

Springer Nature remains neutral with regard to jurisdictional claims in published maps and institutional affiliations. 